\title{
TELEMETRIC SENSORS FOR THE SPACE LIFE SCIENCES
}

\author{
John W. Hines(1), Chris J. Somps(1), Marc Madou(1), Dean C. Jeutter(2), Avtar Singh(3) \\ (1)NASA-Ames Research Center, MS 213-2, Moffett Field, CA 94035-1000, USA \\ John_Hines@qmgate.arc.nasa.gov, Chris_Somps@qmgate.arc.nasa.gov, madsense@aol.com \\ ${ }^{(2)}$ College of Engineering, Marquette University, Milwaukee, WI 53201-1881, USA \\ jeutterd@vms.csd.mu.edu \\ (3)Electrical Engineering, San Jose State University, San Jose, CA 95192-0084, USA \\ asingh@sparta.sjsu.edu
}

\begin{abstract}
Telemetric sensors for monitoring physiological changes in animal models in space are being developed by NASA's Sensors 2000 ! program. The sensors measure a variety of physiological measurands, including temperature, biopotentials, pressure, flow, acceleration, and chemical levels, and transmit these signals from the animals to a remote receiver via a wireless link. Thus physiologic information can be obtained continuously and automatically without animal handling, tethers, or percutaneous leads. We report here on NASA's development and testing of advanced wireless sensor systems for space life sciences research.
\end{abstract}

\section{Introduction}

Monitoring and acquiring physiologic data from unrestrained space flight research subjects is currently intermittent, labor intensive, and heavily biased toward ground-based pre and post flight testing periods. Continuous signals are typically acquired with hardwired sensors and tethered animals ${ }^{1}$. Because future mission durations will be longer the ability to acquire physiologic data from untethered subjects, chronically and automatically during flights, is being requested by the space life sciences research community 2 . In addition, multiple channels, higher bandwidths, and chemical parameters are needed. To meet this need for advanced sensor systems for space life sciences research, NASA's Sensors 2000! program is developing wireless biotelemetry data acquisition systems ${ }^{3}$. We report here on the development of biocompatible sensors, implantable biotelemeters, and radio receivers.

\section{NASA's Sensors 2000! Program}

Sensors 2000! (S2K!) is a NASA Ames Research Center engineering initiative designed to provide biosensor and bioinstrumentation systems expertise to NASA's life sciences space flight programs. The programs include both international and multidisciplinary missions and payloads, including collaborative unmanned flights (Bion-10 \& 11) Space Shuttle flights (Neurolab), and future Space Station flights. S2K! covers the full spectrum of sensor technology applications, ranging from space flight hardware design and fabrication to advanced technology development, transfer and commercialization. Biotelemetry systems and components currently under development by $S 2 \mathrm{~K}$ ! and its university and industry collaborators include an in vivo $\mathrm{pH}$ sensor, a 4 channel, implantable biotelemeter and portable receiver, a DSP-based signal decoder, and a multichannel, microcontroller-based PCM system.

\section{Telemetric Sensors and Systems}

\section{$\mathrm{pH}$ Sensor}

The $\mathrm{pH}$ sensor is based on a polymeric $\mathrm{H}^{+}$-sensitive neutral carrier membrane cast around the tip of a microbore PVC catheter. It consists of two single lumen catheters (physically combined or separated); one lumen each for the reference and pH electrodes (Fig. 1). The membrane composition is high molecular weight poly (vinyl chloride), bis (2-ethyl hexyl) adipate, and potassium tetrakis (4chlorophenyl) borate. Tri-n-dodecylamine is used as the $\mathrm{H}^{+}$ ionophore.

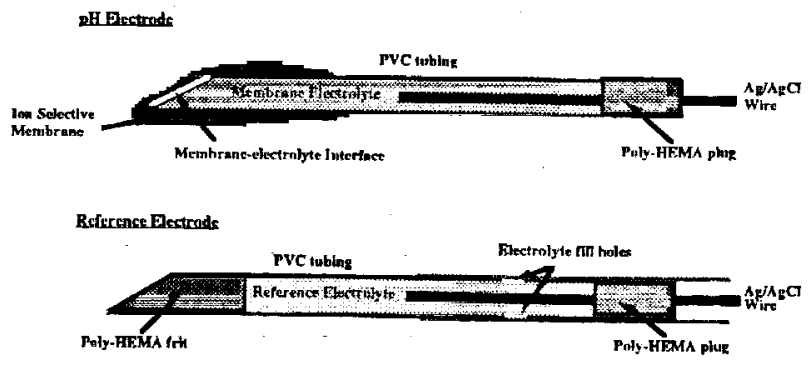

Figure 1: Diagram of the $\mathrm{pH}$ and reference electrodes

Our microbore $\mathrm{pH}$ sensors exhibit response characteristics similar to larger, commercially available $\mathrm{pH}$ electrodes. Baseline drift is on the order of 2-4 mV (.03-.07 pH units) every 24 hours. Sensor sensitivity is in the expected range of $57-60 \mathrm{mV} / \mathrm{pH}$ unit at room temperatures (Theoretical $=59 \mathrm{mV} / \mathrm{pH} @ 25^{\circ} \mathrm{C}$ ), and sensitivity loss is only $1-2 \%$ over a 12 day period. For the particular ionophore used, response linearity breaks down around $\mathrm{pH}$ 5. However, for our applications, the range of interest (6.88.0) falls within the linear range of the sensor. Response 
times are rapid with roughly $95 \%$ of the response achieved within less than 3 seconds. These response characteristics are not notably changed following subcutaneous implantation in rats for up to 12 days.

\section{Biotelemeter \& Receiver}

A totally implantable, digitally encoded biotelemeter for measurement of $\mathrm{pH}$, temperature, and heart rate has been designed, prototyped, and successfully bench tested in conjunction with our microbore $\mathrm{pH}$ electrode. The circuit (Fig. 2) employs a low power A/D converter, a digitalencoding IC (Manchester), and transmits a pulse modulated $455 \mathrm{kHz}$ carrier. It dissipates less than $420 \mu \mathrm{W}$. The device has been miniaturized in thick film hybrid form and will be packaged with a $3 / 4$ Amp-hour battery inside a biocompatible ceramic enclosure. A portable receiver acquires and demodulates the RF carrier, demultiplexes and decodes the 3 data channels, and presents the output to liquid crystal displays (LCD) on the front of the receiver chassis. A prototype receiver has been successfully tested with implants in sheep.

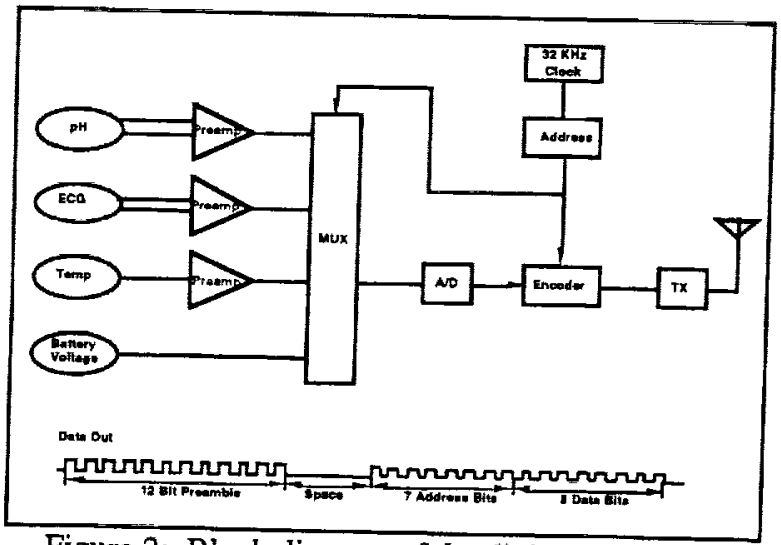

Figure 2: Block diagram of the digitally encoded biotelemeter and sample data packet

\section{DSP Decoder}

We are evaluating the ability of a Digital Signal Processing chip (Texas Instruments TMS320C5X) to decode and display physiologic signals from a variety of commercial and S2K! biotelemeters. Commercially available biotelemeters encode physiologic information using pulse position modulation schemes. The DSP chip is programmed to measure time intervals between pulses and display the decoded physiologic signals on a flat panel LCD screen. The chip can also decode the pulse code modulation (PCM) encoding schemes used with the S2K! biotelemeters The inclusion of a DSP device in a receiver provides signal processing capability to filter, decimate, interpolate, amplify, and attenuate signals, or process them to compute diagnostically useful information such as heart rate from an ECG signal. This technology will be incorporated into our receiver subsystems in order to make them physically smaller, more flexible and adaptable, and consume less spáce and power.

\section{Microcontroller-based Biotelemetry System}

A micropower controller (Motorola MC68HC705 series) is being used to orchestrate the acquisition, processing, and PCM encoding of physiologic signals within a totally implantable bi-directional biotelemeter ${ }^{4}$. The controller also supervises the power utilization of portions of the biotelemeter circuit, and interprets and acts on commands received from an external inlink transmitter. An FSKmodulated RF transmitter transmits the serial PCM code. The transmitted bandwidth is $\sim 300 \mathrm{kHz}$ within the $174-216$ $\mathrm{MHz}$ band, and the center frequency is adjustable via the inlink commands.

\section{Summary}

NASA's Sensors 2000! program, working with University and industry collaborators, is designing, fabricating and testing biotelemetry systems and components including miniaturized, neutral carrier-based $\mathrm{pH}$ sensors, totally implantable digital biotelemeters, digital biotelemetry systems, and portable, easy-to-use receivers and decoders. The technology will be used with animal models on the Space Shuttle and Space Station to further our general understanding of physiological adaptation to the space environment.

\section{References}

[1] Advances in Space Biology and Medicine, Vol. 1, ed. by S.L. Bonting, JAI Press, Inc. Greenwich, Connecticut, 1991.

[2] C.C. Johnson and R.D. Arno, "Life Science Research Objectives and Representative Experiments for the Space Station", NASA TM \#89445, 1989.

[3] J.W. Hines, C.J. Somps, B. Ricks, and L. Kim, "Advanced Biotelemetry Systems for Space Life Sciences: pH Telemetry", In proceedings of the 13th International Symposium on Biotelemetry, Williamsburg, VA, March 26-31, pp 131-137, 1995.

[4] D.C. Jeutter, J.W. Hines, and M.S. Geisler, "Microcontroller based PCM Bio-transceiver System for the ABTS (Advanced Biotelemetry System)", Life sciences and Space Medicine Conference, AIAA/NASA, Houston; AIAA-95-LS-147, 1995. 